\title{
A Study on Labelling of Pythagorean Neutrosophic Fuzzy Graphs
}

Ajay $\mathrm{D}^{*} \mathbf{1}^{1}$, Karthiga $\mathrm{S}^{2}$ and Chellamani $\mathrm{P}^{3}$

Received: 15 February 2021/ Accepted: 15 May 2021/ Published online: 18 June 2021

(C)Sacred Heart Research Publications 2017

\begin{abstract}
Pythagorean neutrosophic fuzzy set comprises elements with dependent membership $(\mu)$, non-membership $(\sigma)$ and independent indeterminacy $(\beta)$ functions with the flexibility $0 \leq \mu^{2}+\beta^{2}+\sigma^{2} \leq 2$. Pythagorean neutrosophic fuzzy graph is a new concept emerged by combining the concept of Pythagorean neutrosophic fuzzy set and fuzzy graph theory. In this paper, the authors present the labelling of Pythagorean neutrosophic fuzzy graphs and investigate their properties.
\end{abstract}

Key words: Pythagorean Neutrosophic, Pythagorean Neutrosophic Fuzzy Graphs, Pythagorean Neutrosophic Fuzzy Labelling Cycle, Pythagorean Neutrosophic Fuzzy Partial Tree.

AMS classification: 03E72, 05C72, 05C78.

\section{Introduction}

As a new emerging mathematical tool, the generalization of a crisp set which has elements with membership grade from interval $[0,1]$ is defined as fuzzy set proposed by Zadeh [1]. This fuzzy set was further developed and extended to intuitionistic set [2], Spherical and Picture fuzzy sets [5, 6]. Smarandache [3] introduced the concept of the Neutrosophic set which is a fuzzy set with the characterization truth, indeterminacy and false as their membership degree. The concept of a single valued neutrosophic set (SVNS) was proposed by Wang et al. [9] as an instance of the neutrosophic set and an extension of intuitionistic fuzzy set with its various properties. SVNSs represent uncertainty, incomplete, imprecise, indeterminate, and inconsistent information. In recent years, researchers have been

\footnotetext{
$1,2,3$ Department of Mathematics, Sacred Heart College (Autonomous), Tirupattur - 635 601, Tamil Nadu, India.
}

Emails: ${ }^{* 1}$ dajaypravin@gmail.com, ${ }^{2}$ karthikasiva1998@gmail.com, ${ }^{3}$ joshmani238@gmail.com 
particularly interested in the neutrosophic set, particularly SVNS. Fuzzy graphs have been combined with neutrosophic sets and labelling of neutrosophic graphs have been defined and their properties have been investigated [12, 13, 14].

To deal with complex imprecision and uncertainty Pythagorean fuzzy sets were proposed by Yager [4, 10, 11] in which the total of the squares of membership and non-membership degrees is between 0 and 1 . Consequently in comparison to intuitionistic fuzzy sets, Pythagorean fuzzy sets account for a greater amount of uncertainty. Smarandache introduced and developed the degree of dependence among components of fuzzy sets and neutrosophic sets. One special case with independent indeterminacy and dependent truth and falsity is chosen out of three membership functions of neutrosophic sets with the constraint that the total of squares of membership, indeterminacy, and non-membership lies between 0 and 2 , and it is known as Pythagorean Neutrosophic set.

Pythagorean neutrosophic fuzzy set [7] is a combination of Pythagorean and neutrosophic set, holding elements with membership and non-membership as dependent component and indeterminacy as independent component with the criteria that the sum of the squares of the membership, non-membership and indeterminacy is between 0 and 2 .

A Pythagorean Neutrosophic fuzzy set with truth and falsity as dependent neutrosophic components [PNFS] on non-empty universe $\mathrm{Y}$ is $P=\left\{\left(e, \mu_{P}(e), \beta_{P}(e), \sigma_{P}(e)\right): e \in Y\right\} \quad$ where $\mu_{P}(e), \beta_{P}(e), \sigma_{P}(e) \in[0,1]$, $0 \leq \mu_{P}(e)^{2}+\beta_{P}(e)^{2}+\sigma_{P}(e)^{2} \leq 2, \forall e \in Y . \mu_{P}(e), \beta_{P}(e), \sigma_{P}(e)$ are the degrees of membership, indeterminacy and non-membership functions respectively.

Pythagorean neutrosophic fuzzy graph is a fusion of Pythagorean neutrosophic fuzzy set and fuzzy graph theory. Ajay and Chellamani [8] proposed the new idea of Pythagorean neutrosophic fuzzy graphs along with few basic properties of the Pythagorean Neutrosophic fuzzy graphs.

Pythagorean Neutrosophic Fuzzy Graph (PNFG) is $G=(V, E)$, where $V=\left\{v_{1}, v_{2}, \ldots, v_{n}\right\}$ such that $\mu_{1}, \beta_{1}$ and $\sigma_{1}$ from $V$ to $[0,1]$ with $0 \leq \mu_{1}^{2}\left(v_{i}\right)+\beta_{1}^{2}\left(v_{i}\right)+\sigma_{1}^{2}\left(v_{i}\right) \leq 2 \forall v_{i}$ in $V$ signifies membership, indeterminacy and non-membership functions correspondingly and $E \subseteq V \times V$ where $\mu_{2}, \beta_{2}, \sigma_{2}$ from $V \times V$ to $[0,1]$ such that $\mu_{2}\left(v_{i} v_{j}\right) \leq \mu_{1}\left(v_{i}\right) \wedge \mu_{1}\left(v_{j}\right), \beta_{2}\left(v_{i} v_{j}\right) \leq \beta_{1}\left(v_{i}\right) \wedge \beta_{1}\left(v_{j}\right)$, $\sigma_{2}\left(v_{i} v_{j}\right) \leq \sigma_{1}\left(v_{i}\right) \vee \sigma_{1}\left(v_{j}\right)$ with $0 \leq \mu_{2}^{2}\left(v_{i} v_{j}\right)+\beta_{2}^{2}\left(v_{i} v_{j}\right)+\sigma_{2}^{2}\left(v_{i} v_{j}\right) \leq 2 \quad \forall\left(v_{i} v_{j}\right) \in E$. 
ISSN: 2456-8686, 5(1), 2021:105-116

https://doi.org/10.26524/cm97

\section{Pythagorean Neutrosophic Fuzzy Labelling Graphs}

Definition 2.1 A Pythagorean neutrosophic fuzzy graph $G=(V, \chi, \varpi)$ is said to be a Pythagorean neutrosophic fuzzy labelling graph if $\chi_{1}: V \rightarrow[0,1], \chi_{2}: V \rightarrow[0,1]$, $\chi_{3}: V \rightarrow[0,1]$ and $\varpi_{1}: V \times V \rightarrow[0,1], \varpi_{2}: V \times V \rightarrow[0,1], \varpi_{3}: V \times V \rightarrow[0,1]$ are one to one such that the membership, indeterminacy and non-membership functions of the edges and vertices where $\chi=\left(\chi_{1}, \chi_{2}, \chi_{3}\right)$ and $\varpi=\left(\varpi_{1}, \varpi_{2}, \varpi_{3}\right)$ are distinct such that

$\varpi_{1}\left(\kappa_{i}, \kappa_{i+1}\right)<\chi_{1}\left(\kappa_{i}\right) \wedge \chi_{1}\left(\kappa_{i+1}\right)$

$\varpi_{2}\left(\kappa_{i}, \kappa_{i+1}\right)<\chi_{2}\left(\kappa_{i}\right) \wedge \chi_{2}\left(\kappa_{i+1}\right)$

$\varpi_{3}\left(\kappa_{i}, \kappa_{i+1}\right)<\chi_{3}\left(\kappa_{i}\right) \vee \chi_{3}\left(\kappa_{i+1}\right)$

$0 \leq\left(\varpi_{1}\left(\kappa_{i}, \kappa_{i+1}\right)\right)^{2}+\left(\varpi_{2}\left(\kappa_{i}, \kappa_{i+1}\right)\right)^{2}+\left(\varpi_{3}\left(\kappa_{i}, \kappa_{i+1}\right)\right)^{2} \leq 2$ for each edge $\left(\kappa_{i}, \kappa_{i+1}\right) \in$ $V \times V$.

Note : In the following theorems and results,

- Pythagorean Neutrosophic Fuzzy Labelling graph, Pythagorean neutrosophic fuzzy labelling and Pythagorean neutrosophic fuzzy will be denoted as PNFLG, PNFL and PNF respectively.

- Membership, Indeterminacy and Non-membership function will be denoted respectively as $\mathrm{M}$, I and N-M.

\section{Example 2.2}

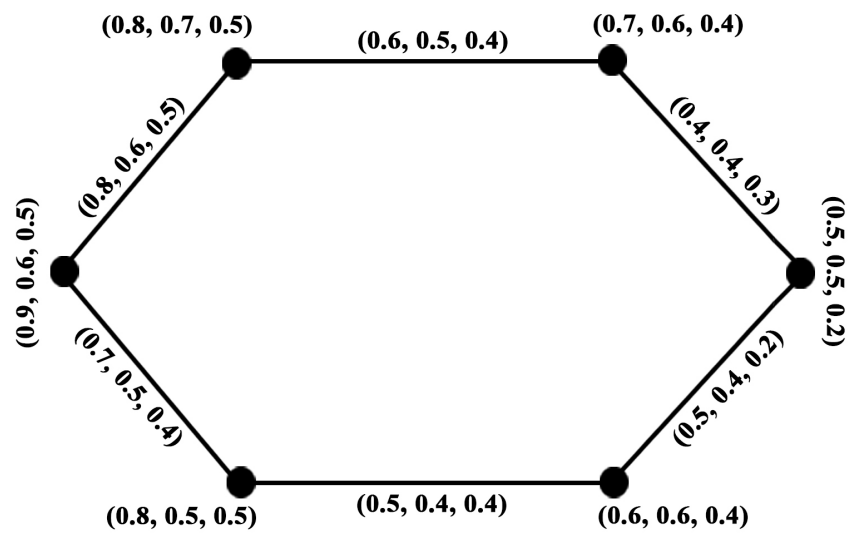

Figure 1: Pythagorean Neutrosophic Fuzzy Graph Labelling 
Definition 2.3 Let $G=(V, \sigma, \mu)$ be a PNFLG $H=(V, \chi, \varpi)$ with $\chi_{1}: V \rightarrow[0,1], \chi_{2}: V \rightarrow[0,1], \chi_{3}: V \rightarrow[0,1]$ and $\varpi_{1}: V \times V \rightarrow[0,1], \varpi_{2}:$ $V \times V \rightarrow[0,1], \varpi_{3}: V \times V \rightarrow[0,1]$ as M,I,N-M of vertices and edges respectively where $\chi=\left(\chi_{1}, \chi_{2}, \chi_{3}\right)$ and $\varpi=\left(\varpi_{1}, \varpi_{2}, \varpi_{3}\right)$ is said to be a Pythagorean neutrosophic fuzzy labelling sub graph of $\mathrm{G}$.

If If $\chi_{1}(\kappa) \leq \sigma_{1}(\kappa), \chi_{2}(\kappa) \leq \sigma_{2}(\kappa)$ and $\chi_{3}(\kappa) \geq \sigma_{3}(\kappa) \forall \kappa \in V$ and $\varpi(\kappa, \varsigma) \leq \mu_{1}(\kappa, \varsigma), \varpi_{2}(\kappa, \varsigma) \leq \mu_{2}(\kappa, \varsigma)$ and $\varpi_{3}(\kappa, \varsigma) \geq \mu_{3}(\kappa, \varsigma) \forall(\kappa, \varsigma) \in V \times V$

Theorem 2.4 If $H=(V, \alpha, \beta)$ with $\alpha=\left(\alpha_{1}, \alpha_{2}, \alpha_{3}\right)$ and $\beta=\left(\beta_{1}, \beta_{2}, \beta_{3}\right)$ is a PNFL subgraph of $G=(V, \chi, \varpi)$ where $\chi=\left(\chi_{1}, \chi_{2}, \chi_{3}\right)$ and $\varpi=\left(\varpi_{1}, \varpi_{2}, \varpi_{3}\right)$ then $\beta_{1}^{\infty}(\kappa, \varsigma) \leq \varpi_{1}^{\infty}(\kappa, \varsigma)$, $\beta_{2}^{\infty}(\kappa, \varsigma) \leq \varpi_{2}^{\infty}(\kappa, \varsigma)$, $\beta_{3}^{\infty}(\kappa, \varsigma) \geq \varpi_{3}^{\infty}(\kappa, \varsigma) \forall \kappa, \varsigma \in V$ where $\beta_{1}^{\infty}, \beta_{2}^{\infty}, \beta_{3}^{\infty}$ and $\varpi_{1}^{\infty}, \varpi_{2}^{\infty}, \varpi_{3}^{\infty}$ are the strength of the Pythagorean neutrosophic fuzzy labelling graph $\mathrm{G}$ and Pythagorean neutrosophic fuzzy labelling sub graph $\mathrm{H}$ of $\mathrm{G}$.

Proof: Let $G=(V, \chi, \varpi)$ be its PNFL sub graph. Let $(\kappa, \varsigma)$ be any PNF path in $\mathrm{G}$ and its strength be $\varpi_{1}^{\infty}(\kappa, \varsigma), \varpi_{2}^{\infty}(\kappa, \varsigma), \varpi_{3}^{\infty}(\kappa, \varsigma)$.

Since $\mathrm{H}$ is a PNFL sub graph of $\mathrm{G}$,

$\alpha_{1}(\kappa) \leq \chi_{1}(\kappa), \beta_{1}(\kappa, \varsigma) \leq \varpi_{1}(\kappa, \varsigma)$,

$\alpha_{2}(\kappa) \leq \chi_{2}(\kappa), \beta_{2}(\kappa, \varsigma) \leq \varpi_{2}(\kappa, \varsigma)$,

$\alpha_{3}(\kappa) \geq \chi_{3}(\kappa), \beta_{3}(\kappa, \varsigma) \geq \varpi_{3}(\kappa, \varsigma) \forall \kappa, \varsigma \in V$ and $(\kappa, \varsigma) \in E \subseteq V \times V$

which implies that,

$\beta_{1}^{\infty}(\kappa, \varsigma) \leq \varpi_{1}^{\infty}(\kappa, \varsigma)$,

$\beta_{2}^{\infty}(\kappa, \varsigma) \leq \varpi_{2}^{\infty}(\kappa, \varsigma)$,

$\beta_{3}^{\infty}(\kappa, \varsigma) \geq \varpi_{3}^{\infty}(\kappa, \varsigma) \forall \kappa, \varsigma \in V$.

Definition 2.5 Let $G=(V, \chi, \varpi)$ be a PNFLG. The strength of the Pythagorean neutrosophic fuzzy path $\mathrm{P}$ of $\mathrm{n}$ edges $e_{i}$ for $\mathrm{i}=1, \ldots . \mathrm{n}$ is denoted by

$P N S(P)=\left(P N S_{1}(P), P N S_{2}(P), P N S_{3}(P)\right)$ and defined as

$P N S_{1}(P)=\min _{1 \leq i \leq n} \varpi_{1}\left(e_{i}\right)$

$P N S_{2}(P)=\min _{1 \leq i \leq n} \varpi_{2}\left(e_{i}\right)$,

$P N S_{3}(P)=\max _{1 \leq i \leq n} \varpi_{3}(e i)$.

Definition 2.6 Let $G=(V, \chi, \varpi)$ be a PNFLG . The Pythagorean neutrosophic 
ISSN: 2456-8686, 5(1), 2021:105-116

https://doi.org/10.26524/cm97

fuzzy strength of connectedness of a duo of vertices $\kappa, \varsigma \in V$, is denoted as

$P N C O N N_{G}(\kappa, \varsigma)=\left(P N C O N N_{1 G}(\kappa, \varsigma), P N C O N N_{2 G}(\kappa, \varsigma), P N C O N N_{3 G}(\kappa, \varsigma)\right)$

and defined by

$P N C O N N_{1 G}(\kappa, \varsigma)=\max \left\{P N S_{1}(P) / P\right.$ is a $\kappa-\varsigma P N F$ path in $\left.G\right\}$;

$P N C O N N_{2 G}(\kappa, \varsigma)=\max \left\{P N S_{2}(P) / P\right.$ is a $\kappa-\varsigma P N F$ path in $\left.G\right\}$;

$P N C O N N_{3 G}(\kappa, \varsigma)=\min \left\{P N S_{3}(P) / P\right.$ is a $\kappa-\varsigma P N F$ path in $\left.G\right\}$.

$P N C O N N_{G}(\kappa, \varsigma)=(0,0,0)$ if the vertices are is defined in $\mathrm{G}$.

Proposition 2.7 Let G be a PNFLG and H a PNFL subgraph of G. Then for every pair of vertices $\kappa, \varsigma \in V$, we take

$P N C O N N_{1 H}(\kappa, \varsigma) \leq P N C O N N_{1 G}(\kappa, \varsigma)$,

$P N C O N N_{2 H}(\kappa, \varsigma) \leq P N C O N N_{2 G}(\kappa, \varsigma)$,

$P N C O N N_{3 H}(\kappa, \varsigma) \geq P N C O N N_{3 G}(\kappa, \varsigma)$.

Definition 2.8 A $\kappa-\varsigma$ Pythagorean neutrosophic fuzzy path in a PNFLG is called the strongest PNF $\kappa-\varsigma$ path if

$P N S_{1}(P)=P N C O N N_{1 G}(\kappa, \varsigma)$,

$P N S_{2}(P)=P N C O N N_{2 G}(\kappa, \varsigma)$,

$P N S_{3}(P)=P N C O N N_{3 G}(\kappa, \varsigma)$.

Definition 2.9 Let G be a PNFLG. A node w is called a pythagorean neutrosophic fuzzy partial cut node (p-cut node) of $\mathrm{G}$ if there exists a pair of nodes $\kappa, \varsigma \in V$ s.t $\kappa \neq \varsigma \neq \xi$ and

$P N C O N N_{1(G-\xi)}(\kappa, \varsigma)<P N C O N N_{1 G}(\kappa, \varsigma)$,

$P N C O N N_{2(G-\xi)}(\kappa, \varsigma)<P N C O N N_{2 G}(\kappa, \varsigma)$,

$P N C O N N_{3(G-\xi)}(\kappa, \varsigma)>P N C O N N_{3 G}(\kappa, \varsigma)$.

A connected PNFLG having no (p-cut node) is called a pythagorean neutrosophic fuzzy partial block.

Definition 2.10 Let G be a PNFLG. An arc $e=(\kappa, \varsigma)$ is called a Pythagorean neutrosophic fuzzy partial bridge

(p- bridge) if

$P N C O N N_{1(G-e)}(\kappa, \varsigma)<P N C O N N_{1 G}(\kappa, \varsigma)$,

$P N C O N N_{2(G-e)}(\kappa, \varsigma)<P N C O N N_{2 G}(\kappa, \varsigma)$,

$\mathrm{PNCONN}_{3(G-e)}(\kappa, \varsigma)>P N C O N N_{3 G}(\kappa, \varsigma)$. 
ISSN: $2456-8686,5(1), 2021: 105-116$

https://doi.org/10.26524/cm97

A ( $\mathrm{p}$ - bridge) is said to be a pythagorean neutrosophic fuzzy partial bond ( $\mathrm{p}$ - bond) if

$P N C O N N_{1(G-e)}(x, y)<P N C O N N_{1 G}(x, y)$,

$P N C O N N_{2(G-e)}(x, y)<P N C O N N_{2 G}(x, y)$

$P N C O N N_{3(G-e)}(x, y)>P N C O N N_{3 G}(x, y)$.

If both $\mathrm{x}$ and $\mathrm{y}$ are different from $\kappa$ and $\varsigma$, the bond is said to be a Pythagorean neutrosophic fuzzy partial cut bond.

\section{Example 2.11}

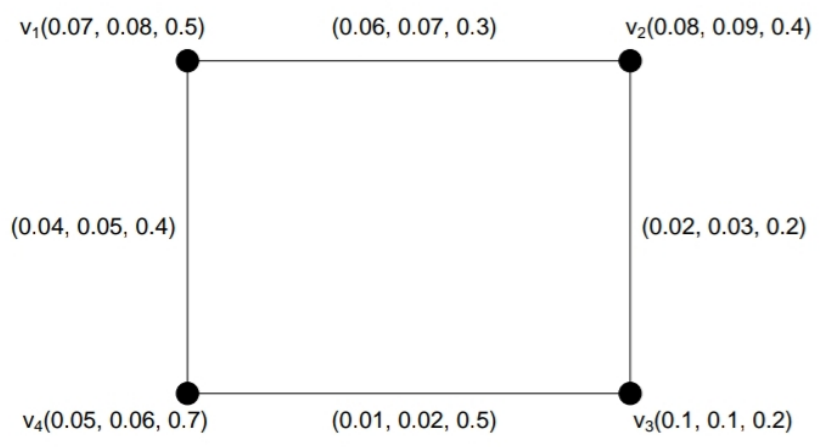

Figure 2: PNFLG with Pythagorean Neutrosophic Fuzzy Partial bridge

Definition 2.12 Let G be a PNFLG and C, a Pythagorean neutrosophic fuzzy cycle in G. Then,

(i) $\mathrm{C}$ is called a strong Pythagorean neutrosophic fuzzy cycle if all pythagorean neutrosophic fuzzy arcs in $\mathrm{C}$ are strong.

(ii) A Pythagorean neutrosophic fuzzy $\operatorname{arc} e=(x, y) \in E$ is called PNF $\alpha$ strong if $P N C O N N_{1(G-e)}(x, y)<\varpi_{1}(\kappa, \varsigma)$,

$P N C O N N_{2(G-e)}(x, y)<\varpi_{2}(\kappa, \varsigma)$,

$P N C O N N_{3(G-e)}(x, y)>\varpi_{3}(\kappa, \varsigma)$;

a $\mathrm{PNF} \delta$ - arc if

$P N C O N N_{1(G-e)}(x, y)>\varpi_{1}(\kappa, \varsigma)$,

$P N C O N N_{2(G-e)}(x, y)>\varpi_{2}(\kappa, \varsigma)$,

$P N C O N N_{3(G-e)}(x, y)<\varpi_{3}(\kappa, \varsigma)$.

(iii) A $\kappa-\varsigma$ Pythagorean neutrosophic fuzzy path $\mathrm{P}$ in $\mathrm{G}$ is named a strong $\kappa-\varsigma$ Pythagorean neutrosophic fuzzy path if all the edges of $\mathrm{P}$ are strong. In particular, 
ISSN: 2456-8686, 5(1), 2021:105-116

https://doi.org/10.26524/cm97

if all the PNF arcs of $\mathrm{P}$ are PNF $\alpha$ - strong, then $\mathrm{P}$ is called $\alpha$ - strong Pythagorean neutrosophic fuzzy path.

Definition 2.13 A PNFLG is named a $\theta$-Pythagorean neutrosophic fuzzy labelling graph if for all pair of nodes $\kappa$ and $\varsigma$, either all strong Pythagorean neutrosophic fuzzy cycles passing through $\kappa$ and $\varsigma$ have the same strength or there is no strong Pythagorean neutrosophic fuzzy cycle passing through $\kappa$ and $\varsigma$.

Proposition 2.14 Let G be a PNFL cycle, then G has exactly one weakest PNF arc.

Proof: Let $G=(V, \chi, \varpi)$ be a PNFL cycle. Let

$$
\begin{aligned}
& \varpi_{1}(\kappa, \varsigma)=\bigwedge_{i=1}^{n} \varpi_{1}\left(\kappa_{i}, \varsigma_{i}\right) \\
& \varpi_{2}(\kappa, \varsigma)=\bigwedge_{i=1}^{n} \varpi_{2}\left(\kappa_{i}, \varsigma_{i}\right) \\
& \varpi_{3}(\kappa, \varsigma)=\bigvee_{i=1}^{n} \varpi_{3}\left(\kappa_{i}, \varsigma_{i}\right)
\end{aligned}
$$

Since G has PNFL, it will have only one PNF arc with $\varpi(\kappa, \varsigma)=\left(\varpi_{1}(\kappa, \varsigma), \varpi_{2}(\kappa, \varsigma)\right.$, $\left.\varpi_{3}(\kappa, \varsigma)\right)$. If we remove $\varpi(\kappa, \varsigma)$ from $\mathrm{G}$, the Pythagorean neutrosophic fuzzy strength of connectedness will not be reduced implying that $\varpi(\kappa, \varsigma)$ is the weakest PNF arc. Hence there exists only one weakest Pythagorean neutrosophic fuzzy arc in any PNFL cycle.

Proposition 2.15 If $\mathrm{G}$ is a PNFL cycle, then it has (n-1) Pythagorean neutrosophic fuzzy bridges

Proof: Let $G=(V, \chi, \varpi)$ be a PNFLG .By previous proposition, $\mathrm{G}$ has only one weakest Pythagorean neutrosophic fuzzy arc. We know that weakest Pythagorean neutrosophic fuzzy edge is not a Pythagorean neutrosophic fuzzy bridge.This implies that the Pythagorean neutrosophic fuzzy strength of the connectedness will be reduced whenever any edge except weakest one is removed. Hence every PNFLG has (n-1) Pythagorean neutrosophic fuzzy bridges.

Proposition 2.16 If $\mathrm{G}$ is a PNFLG, then every Pythagorean neutrosophic fuzzy bridge is strong and vice versa.

Proof: Let $G=(V, \chi, \varpi)$ be a PNFLG with $\mathrm{n}$ nodes. $\mathrm{G}$ has exactly one weakest pythagorean neutrosophic fuzzy arc and has n-1 picture fuzzy bridges. 
ISSN: 2456-8686, 5(1), 2021:105-116

https://doi.org/10.26524/cm97

Our claim is to prove that n-1 Pythagorean neutrosophic fuzzy bridges are strong. Let us choose a Pythagorean neutrosohic fuzzy arc $\kappa_{i}, \kappa_{i+1}$ from n-1 edges.

Since $G$ is a Pythagorean neutrosophic fuzzy labelling cycle, there are two Pythagorean neutrosophic fuzzy paths between the nodes $\kappa_{i}$ and $\kappa_{i+1}$.

That is one Pythagorean neutrosophic fuzzy path with $\varpi\left(\kappa_{i}, \kappa_{i+1}\right)>0$ and the other with

$\varpi\left(\kappa_{i}, \kappa_{i+1}\right)=\left(\varpi_{1}\left(\kappa_{i}, \kappa_{i+1}\right), \varpi_{2}\left(\kappa_{i}, \kappa_{i+1}\right), \varpi_{3}\left(\kappa_{i}, \kappa_{i+1}\right)\right)$

$\varpi\left(\kappa_{i}, \ldots \kappa_{i+n}, \ldots \kappa_{i+1}\right)$

$=\left(\varpi_{1}\left(\kappa_{i}, \ldots \kappa_{i+n}, \ldots \kappa_{i+1}\right), \varpi_{2}\left(\kappa_{i}, \ldots \kappa_{i+n}, \ldots \kappa_{i+1}\right), \varpi_{3}\left(\kappa_{i}, \ldots \kappa_{i+n}, \ldots \kappa_{i+1}\right)\right)$

$>0$

$\varpi^{\infty}\left(\kappa_{i}, \kappa_{i+1}\right)=\varpi\left(\kappa_{i}, \kappa_{i+1}\right)=\left(\varpi_{1}\left(\kappa_{i}, \kappa_{i+1}\right), \varpi_{2}\left(\kappa_{i}, \kappa_{i+1}\right), \varpi_{3}\left(\kappa_{i}, \kappa_{i+1}\right)\right)$

which implies that $\left(\kappa_{i}, \kappa_{i+1}\right)$ is a strong Pythagorean neutrosophic fuzzy arc. Repeating this argument for all (n-1) edges, we obtain that every Pythagorean neutrosophic fuzzy bridge is strong. The converse of the theorem is obvious.

Proposition 2.17 Let G be a PNFLG. Then G has at least one Pythagorean neutrosophic fuzzy bridge.

Proof: Let $G=(V, \chi, \varpi)$ be a PNFLG. Choose an edge $(\kappa, \varsigma)$ s.t $\varpi^{\prime}(\kappa, \varsigma)=$ $\left(\varpi_{1}(\kappa, \varsigma), \varpi_{2}(\kappa, \varsigma), \varpi_{3}(\kappa, \varsigma)\right)$ is the maximum in the set of all values of $\varpi^{\prime}\left(\kappa_{i}, \varsigma_{i}\right)=$ $\left(\varpi_{1}\left(\kappa_{i}, \varsigma_{i}\right), \varpi_{2}\left(\kappa_{i}, \varsigma_{i}\right), \varpi_{3}\left(\kappa_{i}, \varsigma_{i}\right)\right)>0$ and there exists some edge (u,v) such that $\varpi^{\prime}(u, v)<\varpi^{\prime}(\kappa, \varsigma)$. Now we claim $\varpi^{\prime}(\kappa, \varsigma)$ is a Pythagorean neutrosophic fuzzy bridge. From $\mathrm{G}$ if the edge $(\kappa, \varsigma)$ is removed, then $\mathrm{H}$ is a Pythagorean neutrosophic fuzzy subgraph, thus we have $\varpi_{H}(\kappa, \varsigma)<\varpi^{\prime}(\kappa, \varsigma)$. Therefore $(\kappa, \varsigma)$ is a Pythagorean neutrosophic fuzzy bridge.

Proposition 2.18 If $\mathrm{G}$ is a connected PNFLG then there exists a strong Pythagorean neutrosophic fuzzy path between any pair of nodes.

Proof: Let $G=(V, \chi, \varpi)$ be a connected PNFLG and let $(\kappa, \varsigma)$ be any pair of nodes. This implies $(\kappa, \varsigma)=\left(\varpi_{1}(\kappa, \varsigma), \varpi_{2}(\kappa, \varsigma), \varpi_{3}(\kappa, \varsigma)\right)>0$. Now choose any edge $(\kappa, \xi)$ in $(\kappa, \varsigma)$. If $\varpi(\kappa, \xi)=\varpi^{\prime}(\kappa, \xi)$, then it is Pythagorean neutrosophic fuzzy strong. Otherwise choose any other edge, say $(\kappa, \omega)$, which satisfies $\varpi(\kappa, \omega)=\varpi^{\prime}(\kappa, \omega)$. By repeating this process, we can find a Pythagorean neutrosophic fuzzy path in $(\kappa, \varsigma)$ in which all Pythagorean neutrosophic fuzzy arcs are strong.

Proposition 2.19 Every PNFLG has at least one weakest Pythagorean neutrosophic fuzzy arc. 
Proof: Let $\mathrm{G}$ be a PNFLG and let $(\kappa, \varsigma)$ be an edge of $\mathrm{G}$ such that the membership, indeterminacy and non-memberships of this edge is maximum, maximum and minimum than all other edges of the Pythagorean neutrosophic fuzzy graph. If this edge is removed from $G$, it does reduce the Pythagorean neutrosophic fuzzy strength of any Pythagorean neutrosophic fuzzy path. That is, after its removal, M, I and N-M of $(\kappa, \varsigma)$ in $\mathrm{G}$ is less, less and greater than the $\mathrm{M}$, I and $\mathrm{N}-\mathrm{M}$ of $(\kappa, \varsigma)$ in the Pythagorean neutrosophic fuzzy labelling subgraph. This implies that the edge is neither a Pythagorean neutrosophic fuzzy bridge nor a strong Pythagorean neutrosophic fuzzy arc. Therefore, this must be the weakest Pythagorean neutrosophic fuzzy arc. Now we define a Pythagorean neutrosophic fuzzy labelling tree along with some of its properties.

Definition 2.20 A PNFL graph $G=(V, \chi, \varpi)$ is named a PNFL tree, if it has PNFL and a PNF spanning sub graph $F=(V, \vartheta, \rho)$ which is a Pythagorean neutrosophic fuzzy tree, where for all PNF arcs $(\kappa, \varsigma)$ not in $\mathrm{F}$,

$\varpi_{1}(\kappa, \varsigma)<\rho_{1}^{\infty}(\kappa, \varsigma)$,

$\varpi_{2}(\kappa, \varsigma)<\rho_{2}^{\infty}(\kappa, \varsigma)$,

$\varpi_{3}(\kappa, \varsigma)>\rho_{3}^{\infty}(\kappa, \varsigma)$.

Theorem 2.21 If G is a PNFL tree, then the arcs of PNF spanning sub graph $\mathrm{F}$ are PNF bridges of $\mathrm{G}$.

Proof: Let $G=(V, \chi, \varpi)$ be a PNFL tree and $F=(V, v, \rho)$ be its PNF spanning sub graph. Let $(\kappa, \varsigma)$ be an arc in $\mathrm{F}$. Then

$\rho_{1}^{\infty}(\kappa, \varsigma)<\varpi_{1}(\kappa, \varsigma) \leq \varpi_{1}^{\infty}(\kappa, \varsigma)$,

$\rho_{2}^{\infty}(\kappa, \varsigma)<\varpi_{2}(\kappa, \varsigma) \leq \varpi_{2}^{\infty}(\kappa, \varsigma)$ and

$\rho_{3}^{\infty}(\kappa, \varsigma)>\varpi_{3}(\kappa, \varsigma) \geq \varpi_{3}^{\infty}(\kappa, \varsigma)$,

which implies that the Pythagorean neutrosophic fuzzy arc $(\kappa, \varsigma)$ is a PNF bridge of G. Because the Pythagorean neutrosophic fuzzy arc $(\kappa, \varsigma)$ is random, the Pythagorean neutrosophic fuzzy arcs of $\mathrm{F}$ are PNF bridges of $\mathrm{G}$.

Theorem 2.22 If there is at most one stronger Pythagorean neutrosophic fuzzy path among any two vertices of PNFLG, then the PNFLG should be a Pythagorean neutrosophic fuzzy partial forest.

Proof: Let us suppose that the PNFLG is not a Pythagorean neutrosophic fuzzy partial forest. Then there will be a Pythagorean neutrosophic fuzzy cycle $\mathrm{C}$ in $\mathrm{G}$ 
ISSN: 2456-8686, 5(1), 2021:105-116

https://doi.org/10.26524/cm97

with the criteria that

$P N C O N N_{1 G}(\kappa, \varsigma) \leq \varpi_{1}(\kappa, \varsigma)$,

$P N C O N N_{2 G}(\kappa, \varsigma) \leq \varpi_{2}(\kappa, \varsigma)$,

$\operatorname{PNCONN}_{3 G}(\kappa, \varsigma) \geq \varpi_{3}(\kappa, \varsigma)$ for all edges $(\kappa, \varsigma)$ of $\mathrm{C}$.

Thus, this edge is the strongest Pythagorean neutrosophic fuzzy path from $\kappa$ to $\varsigma$. But if we pick this edge to be the frailest edge of $\mathrm{C}$, then the other edges of $\mathrm{C}$ will also be the strongest Pythagorean neutrosophic fuzzy path from $\kappa$ to $\varsigma$, which is a contradiction.

Theorem 2.23 If $\mathrm{G}$ is a Pythagorean neutrosophic fuzzy partial tree but not a Pythagorean neutrosophic fuzzy tree, then there exists at least one edge for which the membership of edge is less than the strength of connectedness of that edge.

Proof: Let $G=(V, \chi, \varpi)$ be a Pythagorean neutrosophic fuzzy partial tree .

Then by the definition of Pythagorean neutrosophic fuzzy partial tree there is a Pythagorean neutrosophic fuzzy spanning tree $\mathrm{F}$ of $\mathrm{G}$ with the condition

$\varpi_{1}(\kappa, \varsigma)<P N C O N N_{1 G}(\kappa, \varsigma)$,

$\varpi_{2}(\kappa, \varsigma)<P N C O N N_{2 G}(\kappa, \varsigma)$,

$\varpi_{3}(\kappa, \varsigma)>P N C O N N_{3 G}(\kappa, \varsigma)$ for edges $(\kappa, \varsigma)$ in $\mathrm{G}$ not in $\mathrm{F}$.

Since $G$ is not a Pythagorean neutrosophic fuzzy tree, there is at least one such Pythagorean neutrosophic fuzzy arc and thus the theorem is proved.

Theorem 2.24 Let $\mathrm{G}$ be a Pythagorean neutrosophic fuzzy partial tree and $\mathrm{F}$ the spanning Pythagorean neutrosophic fuzzy tree.Then the arcs of $\mathrm{F}$ are the Pythagorean neutrosophic fuzzy partial bridges of $\mathrm{G}$.

Proof: Let $G=(V, \chi, \varpi)$ be a Pythagorean neutrosophic fuzzy partial tree and $(\kappa, \varsigma)$ an arc in F. Since F is a Pythagorean neutrosophic fuzzy spanning tree of $G$, this arc is a unique Pythagorean neutrosophic fuzzy path from $\kappa$ to $\varsigma$ in $\mathrm{F}$. The result is trivial if there is no other Pythagorean neutrosophic fuzzy path in $G$ from $\kappa$ to $\varsigma$. If there is a Pythagorean neutrosophic fuzzy path from $\kappa$ to $\varsigma$ in $\mathrm{G}$, then the Pythagorean neutrosophic fuzzy path will definitely have a Pythagorean neutrosophic fuzzy arc $(\kappa, \varsigma)$ such that

$P N C O N N_{1 G}(\kappa, \varsigma)>\varpi_{1}(\kappa, \varsigma)$,

$P N C O N N_{2 G}(\kappa, \varsigma)>\varpi_{2}(\kappa, \varsigma)$,

$P N C O N N_{3 G}(\kappa, \varsigma)<\varpi_{3}(\kappa, \varsigma)$

Then it is shown that $(\kappa, \varsigma)$ is not a frailest edge of any Pythagorean neutrosophic fuzzy cycle in $\mathrm{G}$. Thus $(\kappa, \varsigma)$ is a Pythagorean neutrosophic fuzzy partial bridge. 
ISSN: 2456-8686, 5(1), 2021:105-116

https://doi.org/10.26524/cm97

\section{Conclusions}

Pythagorean Neutrosophic fuzzy graph is a new concept which is a combo of Pythagorean Neutrosophic fuzzy set and fuzzy graph theory. In this paper, we have introduced Pythagorean neutrosophic fuzzy labelling of graphs. And also investigated some important properties of Pythagorean neutrosophic fuzzy labelling graphs like Pythagorean neutrosophic fuzzy labelling cycle and PNFL tree. Furthermore, we would make a study on Pythagorean neutrosophic fuzzy magic labelling of graphs and investigate the properties.

\section{References}

[1] Zadeh L A, Fuzzy Sets. Inform. and Control, 8, 338 - 353, (1965).

[2] Atanassov K, Intuitionistic Fuzzy Sets. Fuzzy Sets and Systems, 20, 87 - 96, (1986).

[3] Smarandache F, A unifying field of logics. Neutrosophy: neutrosophic probability, set and logic. American Research Press, 1 - 141, (1998).

[4] Yager R R, Pythagorean Fuzzy Subsets. In:Proc Joint IFSA World Congress and NAFIPS Annual Meeting, Edmonton, Canada, 57 - 61, (2013).

[5] Cuong B C, Kreinovich V, Picture fuzzy sets. Journal of Computer Science and Cybernetics, 30, 409 - 420, (2014).

[6] Kutlu Gündoğdu F, Kahraman C, Spherical fuzzy sets and spherical fuzzy TOPSIS method. Journal of Intelligent and Fuzzy Systems, 36, 337 - 352, (2019).

[7] Jansi R, Mohana R K, Smarandache F, Correlation Measure for Pythagorean Neutrosophic Fuzzy Sets with T and F as Dependent Neutrosophic Components Correlation Measure for Pythagorean Neutrosophic Fuzzy Sets with T and F as Dependent Neutrosophic Components. Neutrosophic Sets and Systems, 30(1) 202 - 212, (2019).

[8] Ajay D, Chellamani P, Pythagorean Neutrosophic Fuzzy Graphs. International Journal of Neutrosophic Science, 11, 108 - 114, (2020).

[9] Wang H, Smarandache F, Zhang Y Q, Sunderraman R, Single valued neutrosophic sets. Multispace and Multistructure, 4, 410 - 413, (2010). 
ISSN: 2456-8686, 5(1), 2021:105-116

https://doi.org/10.26524/cm97

[10] Yager R R, Abbasov A M, Pythagorean membership grades, complex numbers and decision making. Int. J. Intell. Syst., 28, 436 - 452, (2013).

[11] Yager R R, Pythagorean membership grades in multi-criteria decision making. IEEE Trans. Fuzzy Syst., 22, 958 - 965, (2014).

[12] Ajay D, Charisma J J, Chellamani P, Fuzzy Magic and Bi-magic Labelling of Intuitionistic Path Graph. International Journal of Recent Technology and Engineering, 8(4), 11508-11512, (2019).

[13] Ajay D, Chellamani P, Fuzzy magic labelling of Neutrosophic path and star graph. Advances in Mathematics: Scientific Journal, 9(8), 6059-6070, (2020).

[14] Devaraj A, Picture fuzzy labelling graphs with an application. Annals of Optimization Theory and Practice, 3(3), 117-134, (2020). 